\title{
Changes in the genetic variance of a simulated dairy cattle population in the presence of a major gene
}

Ghobad Asgari Jaffarabadi

Islamic Azad University of Varamin, Varamin, Tehran, Islamic Republic of Iran

Email: ghobad@gmail.com

Introduction Intense selection for quantitative traits has recently led to the unexpected appearance of new genes with major effects. Some examples of major genes affecting dairy traits are kappa-casein gene which has a significant effect on milk yield and milk protein. When having genes with large effects on the trait, in addition to polygenes, the truncation selection of animals with best polygenic and major breeding values affects the whole variances of the trait in population. In this research our aim was to study the rate of changes in different genetic and phenotypic variances under different levels of major gene contribution having the same parameters which may be seen in dairy cattle populations.

Materials and methods Stochastic simulation was applied to generate populations with 20 replicates. Breeding values of major gene were simulated depending on allelic frequencies and genotype of each individual. Phenotypic value for each animal was made using a model consisting fixed effects, polygenic and major gene breeding values and residual random effect. The inclusion of fixed effects in the model was same as the method of Abdel-Azim and Freeman (2002). The total phenotypic value was partitioned to $40 \%$ due to fixed factors and $60 \%$ due to random factors (Kuhn et al., 1994). Of the $40 \%$, due to fixed components, the herd effect accounted for $22 \%$ and the year effect for $6 \%$. Other fixed factors accounted for the remaining $12 \%$. The trait of interest was milk yield in cows having the phenotypic variance of 1200,000 and mean of $6500 \mathrm{~kg}$. The base population was consisted of 5000 females and 50 males with overlapping generations. Maximum of five parities was assumed for each dam. The simulation was applied for 20 years of selection with three different polygenic heritabilities of $0.1,0.3$ and 0.5 respectively. For the major gene, there were three different initial frequencies of favourable allele $(\mathrm{p}=0.1, \mathrm{p}=0.3$ and $\mathrm{p}=0.6)$, three different modes of inheritance including co-dominance, partial and complete dominance $(\mathrm{d}=0, \mathrm{~d}=0.5$ and $\mathrm{d}=1$ additive value) and three different values for the magnitude of additive effect ( $\mathrm{a}=0.1$, $\mathrm{a}=0.5$ and $\mathrm{a}=1$ phenotypic standard deviation). So there exist 81 different combinations for our simulation. The contribution of major gene was calculated as the ratio of genetic variance caused by major gene to total additive genetic variance. For each level of contribution the four variances (total breeding values, major gene, polygenic and phenotypic variance) were computed. The rate of changes in these four variances was calculated as the slope of regression line when each variance was regressed over years of simulation. Then, these coefficients were plotted against levels of contribution for each of four variances. Again new linear coefficients were found for variances, showing the trend of increase or decrease in rate of their changes. The linear and quadratic regression coefficients of year on each variance were derived under each combination and tested using T-test procedure.

Results There were different levels of major gene contribution ranging from $0.36 \%$ to $84 \%$. As it is shown in Table 1 , as level of contribution increases, the rate of changes in major gene variance reduces significantly. Results show that the rate of changes in polygenic variance does not differ significantly with the changes in levels of major genes contribution. For the total additive variance, the rate of changes for different levels of contribution is much denser in comparison to major gene variance. Also it can be shown that the rate of changes in phenotypic variance follows the same pattern of total additive variance. The reduction in the rate of changes and the divergence happens in the same way that total additive variance did.

Table 1 Linear regression coefficients of rate of variance changes on levels of major gene contribution

\begin{tabular}{ll}
\hline \hline Variance & Standardized linear regression coefficients \\
\hline Total breeding values & $-0.571^{*}$ \\
Major gene & $-0.319^{*}$ \\
Polygenic & $-0.169^{\text {n.s }}$ \\
Phenotypic & $-0.574^{*}$ \\
\hline \hline
\end{tabular}

Conclusion In general, as the level of major gene contribution in total additive variance increases, the rate of changes in genetic and phenotypic variances decreases. This means that at the low levels of contribution we may expect fast changes in genetic variances, but by an increase in frequency of favourable allele, level of dominance and magnitude of additive effect which leads to increase of major gene variance, changes in variances occur more slowly. So when there is a major gene in a population of dairy cattle with overlapping generations, the breeder may expect to have rapid changes in variances at low levels of contribution and so choose the best policy of utilizing these favourable variations as a tool of selection in his herd. But with increase in frequency of favourable allele of major gene which tries to reach to fixation and also increase of gametic phase disequilibrium, this variation begins to reduce but not as fast as it happened in early years of selection.

\section{References}

Abdel-Azim, G., and Freeman, A.E. 2002. Superiority of QTL-Assisted selection in Dairy Cattle Breeding Schemes. Journal of Dairy Science 85, 1869-1880.

Kuhn, M. T., Boettcher, P. J. and Freeman, A. E. 1994. Potential biases in predicted transmitting abilities of females from preferential treatment. Journal of Dairy Science 77, 2428-2437. 